\title{
KOSZUL DUALITY FOR DIOPERADS
}

\author{
WeE Liang Gan
}

\begin{abstract}
We introduce the notion of a dioperad to describe certain operations with multiple inputs and multiple outputs. The framework of Koszul duality for operads is generalized to dioperads. We show that the Lie bialgebra dioperad is Koszul.
\end{abstract}

\section{Introduction}

The current interest in the understanding of various algebraic structures using operads is partly due to the theory of Koszul duality for operads, which was developed by Ginzburg-Kapranov in [GiK]; see eg. [Ka] or [L] for surveys. However, algebraic structures such as bialgebras and Lie bialgebras, which involve both multiplication and comultiplication, or bracket and cobracket, are defined using PROP's (cf. [Ad]) rather than operads. Inspired by the theory of string topology of Chas-Sullivan (cf. [ChS], [Ch], [Tr]), Victor Ginzburg suggested to the author that there should be a theory of Koszul duality for PROP's. This paper results from the attempt to develop such a theory. More precisely, we introduce the notion of a dioperad, which can be used to describe certain operations with multiple inputs and multiple outputs. We show that one can set up a theory of Koszul duality for dioperads, and we prove that the dioperad associated to Lie bialgebra is Koszul.

Let us explain how dioperads arise. Suppose $\mathcal{Q}=\{\mathcal{Q}(m, n)\}$ is a PROP defined by some generators and relations. We think of an element in $\mathcal{Q}(m, n)$ as an operation obtained by compositions of the generators according to a "flow chart" with $n$ inputs and $m$ outputs. Assuming that the defining relations between the generators of $\mathcal{Q}$ are expressed by flow charts which are trees, there is a subspace $\mathcal{P}(m, n)$ of $\mathcal{Q}(m, n)$ consisting of those operations obtained from the flow charts which are trees. The collection $\mathcal{P}=\{\mathcal{P}(m, n)\}$ is precisely the dioperad with the same generators and relations as $\mathcal{Q}$. Since the defining relations of $Q$ are expressed by trees, no essential information is lost by restricting our attention to $\mathcal{P}$. If $f$ and $g$ are operations in $\mathcal{P}$, then by substituition of the $j$-th output of $g$ into the $i$-th input of $f$, we get another operation $f_{i} \circ_{j} g$ which is still in $\mathcal{P}$. Pictorially, this just means that if we join a root of a tree to a leaf of another tree, we still get a tree.

The assumption that the defining relations between the generators of $Q$ are expressed by trees is satisfied, for example, by the Lie bialgebra PROP, but

Received January 30, 2002. 
not by the bialgebra PROP; see eg. [ES] p.19 and p.70. Thus, Lie bialgebras can be understood in the framework of dioperads, but bialgebras cannot. The above assumption is motivated by the question of what is a "quadratic" PROP. It seems an answer (suggested by the fact that the differential in Kontsevich's graph complex $[\mathrm{Ko}]$ is induced by edge contractions) is that the defining relations between the generators should be express by graphs with precisely one internal edge; cf. also $[\mathrm{Ge}] \S 4.8$. However, a graph with one internal edge is necessarily a tree.

The paper is organized as follows. In $\S 1$, we give the definition of a dioperad and other generalities. In $\S 2$, we define the notion of a quadratic dioperad, its quadratic dual, and introduce our main example of Lie bialgebra dioperad. In $\S 3$, we define the cobar dual of a dioperad. A quadratic dioperad is Koszul if its cobar dual is quasi-isomorphic to its quadratic dual. In $\S 4$, we prove a proposition to be used later in $\S 5$. This proposition is a generalization of a result of ShniderVan Osdol [SVO]. In $\S 5$, we prove that Koszulity of a quadratic dioperad is equivalent to exactness of certain Koszul complexes. In the case of operads, this is due to Ginzburg-Kapranov, with a different proof by Shnider-Van Osdol. The Koszulity of the Lie bialgebra dioperad follows from this and an adaptation of results of Markl [M2].

\section{Dioperads}

1.1. We give in this subsection the definition of dioperad which is similar to the definition of operad in [M1]; cf. also [M].

Let $\mathcal{C}$ be the symmetric monoidal category of finite dimensional differential $\mathbb{Z}$-graded super vector spaces over a field $\mathbb{k}$ of characteristic 0 , and let $\mathcal{H}$ om be the internal hom functor of $\mathcal{C}$. Let $\mathbb{S}_{n}$ denote the automorphism group of $\{1, \ldots, n\}$. If $m=m_{1}+\cdots+m_{n}$ is an ordered partition and $\sigma \in \mathbb{S}_{n}$, then the block permutation $\sigma_{m_{1}, \ldots, m_{n}} \in \mathbb{S}_{m}$ is the permutation that acts on $\{1, \ldots, m\}$ by permuting $n$ intervals of lengths $m_{1}, \ldots, m_{n}$ in the same way that $\sigma$ permutes $1, \ldots, n$. If $\sigma_{1} \in \mathbb{S}_{n_{1}}, \sigma_{2} \in \mathbb{S}_{n_{2}}$ and $i \in\left\{1, \ldots, n_{1}\right\}$, then define $\sigma_{1} \circ_{i} \sigma_{2} \in$ $\mathbb{S}_{n_{1}+n_{2}-1}$ by

$$
\sigma_{1} \circ_{i} \sigma_{2}:=\left(\sigma_{1}\right)_{1, \ldots, 1, n_{2}, 1 \ldots, 1} \circ\left(\operatorname{Id} \times \cdots \times \sigma_{2} \times \cdots \times \operatorname{Id}\right),
$$

where $\sigma_{2}$ is at the $i$-th place. (See eg. [MSS] Definition 1.2 or [SVO] p.387. )

A dioperad $\mathcal{P}$ in $\mathcal{C}$ consists of data:

(i) objects $\mathcal{P}(m, n)$, equipped with a $\left(\mathbb{S}_{m}, \mathbb{S}_{n}\right)$-bimodule structure, for each ordered pair of positive integers $m, n$;

(ii) morphisms ${ }_{i} \circ_{j}: \mathcal{P}\left(m_{1}, n_{1}\right) \otimes \mathcal{P}\left(m_{2}, n_{2}\right) \rightarrow \mathcal{P}\left(m_{1}+m_{2}-1, n_{1}+n_{2}-1\right)$ for each $m_{1}, m_{2}, n_{1}, n_{2} \geq 1$ and $1 \leq i \leq n_{1}, 1 \leq j \leq m_{2}$;

(iii) a morphism $\eta: \mathbb{k} \rightarrow \mathcal{P}(1,1)$ such that

$$
1 \circ_{i}(\eta \otimes \mathrm{Id}): \mathbb{k} \otimes \mathcal{P}(m, n) \stackrel{\sim}{\rightarrow} \mathcal{P}(m, n)
$$


and

$$
j \circ_{1}(\mathrm{Id} \otimes \eta): \mathcal{P}(m, n) \otimes \mathbb{k} \stackrel{\sim}{\rightarrow} \mathcal{P}(m, n)
$$

are the canonical isomorphisms for all $m, n \geq 1$ and $1 \leq i \leq m, 1 \leq j \leq n$.

These data are required to satisfy the following associativity and equivariance conditions:

(a) for all $m_{1}, n_{1}, m_{2}, n_{2}, m_{3}, n_{3} \geq 1$ and $1 \leq i \leq n_{1}+n_{2}-1,1 \leq j \leq m_{3}$, $1 \leq k \leq n_{1}, 1 \leq l \leq m_{2}$, the morphism

$$
\begin{aligned}
i{ }_{i}\left({ }_{k} \circ_{l} \otimes \mathrm{Id}\right): \mathcal{P}\left(m_{1}, n_{1}\right) \otimes \mathcal{P}( & \left.m_{2}, n_{2}\right) \otimes \mathcal{P}\left(m_{3}, n_{3}\right) \\
& \rightarrow \mathcal{P}\left(m_{1}+m_{2}+m_{3}-2, n_{1}+n_{2}+n_{3}-2\right)
\end{aligned}
$$

is equal to

$$
\begin{cases}(\sigma, 1)\left(k+n_{3}-1 \circ_{l}\right)\left({ }_{i} \circ_{j} \otimes \mathrm{Id}\right)(\mathrm{Id} \otimes \tau) & \text { if } i \leq k-1 \\ k \circ_{j+l-1}\left(\mathrm{Id} \otimes_{i-k+1} \circ_{j}\right) & \text { if } k \leq i \leq k+n_{2}-1 \\ (\sigma, 1)\left({ }_{k} \circ_{l}\right)\left({ }_{i-n_{2}+1} \circ_{j} \otimes \mathrm{Id}\right)(\mathrm{Id} \otimes \tau) & \text { if } k+n_{2} \leq i\end{cases}
$$

where

$$
\tau: \mathcal{P}\left(m_{2}, n_{2}\right) \otimes \mathcal{P}\left(m_{3}, n_{3}\right) \stackrel{\sim}{\rightarrow} \mathcal{P}\left(m_{3}, n_{3}\right) \otimes \mathcal{P}\left(m_{2}, n_{2}\right)
$$

is the symmetry isomorphism, and $\sigma \in \mathbb{S}_{m_{1}+m_{2}+m_{3}-2}$ is the block permutation

$$
((12)(45))_{l-1, j-1, m_{1}, m_{3}-j, m_{2}-l} \text {. }
$$

(b) for all $m_{1}, n_{1}, m_{2}, n_{2}, m_{3}, n_{3} \geq 1$ and $1 \leq i \leq n_{1}, 1 \leq j \leq m_{2}+m_{3}-1$, $1 \leq k \leq n_{2}, 1 \leq l \leq m_{3}$, the morphism

$$
\begin{aligned}
i \circ_{j}\left(\operatorname{Id} \otimes_{k} \circ_{l}\right): \mathcal{P}\left(m_{1}, n_{1}\right) \otimes & \mathcal{P}\left(m_{2}, n_{2}\right) \otimes \mathcal{P}\left(m_{3}, n_{3}\right) \\
& \rightarrow \mathcal{P}\left(m_{1}+m_{2}+m_{3}-2, n_{1}+n_{2}+n_{3}-2\right)
\end{aligned}
$$

is equal to

$$
\begin{cases}(1, \sigma)\left({ }_{k} \circ_{l+m_{1}-1}\right)\left(\mathrm{Id} \otimes_{i} \circ_{j}\right)(\tau \otimes \mathrm{Id}) & \text { if } j \leq l-1 \\ k+i-1 \circ_{l}\left({ }_{i} \circ_{j-l+1} \otimes \mathrm{Id}\right) & \text { if } l \leq j \leq l+m_{2}-1 \\ (1, \sigma)\left({ }_{k} \circ_{l}\right)\left(\mathrm{Id} \otimes_{i} \circ_{j-m_{2}+1}\right)(\tau \otimes \mathrm{Id}) & \text { if } l+m_{2} \leq j\end{cases}
$$

where

$$
\tau: \mathcal{P}\left(m_{1}, n_{1}\right) \otimes \mathcal{P}\left(m_{2}, n_{2}\right) \stackrel{\sim}{\rightarrow} \mathcal{P}\left(m_{2}, n_{2}\right) \otimes \mathcal{P}\left(m_{1}, n_{1}\right)
$$

is the symmetry isomorphism, and $\sigma \in \mathbb{S}_{n_{1}+n_{2}+n_{3}-2}$ is the block permutation

$$
((12)(45))_{i-1, k-1, n_{3}, n_{2}-k, n_{1}-i} \text {. }
$$

(c) for all $m_{1}, n_{1}, m_{2}, n_{2} \geq 1,1 \leq i \leq n_{1}, 1 \leq j \leq m_{2}$, and $\pi_{1} \in \mathbb{S}_{m_{1}}, \sigma_{1} \in \mathbb{S}_{n_{1}}$, $\pi_{2} \in \mathbb{S}_{m_{2}}, \sigma_{2} \in \mathbb{S}_{n_{2}}$, the morphism

$$
\begin{aligned}
i{ }_{i}\left(\left(\pi_{1}, \sigma_{1}\right) \otimes\left(\pi_{2}, \sigma_{2}\right)\right): \mathcal{P}\left(m_{1}, n_{1}\right) \otimes \mathcal{P}\left(m_{2}, n_{2}\right) & \\
& \rightarrow \mathcal{P}\left(m_{1}+m_{2}-1, n_{1}+n_{2}-1\right)
\end{aligned}
$$


is equal to

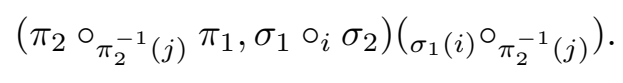

\section{Remarks.}

(1) We shall regard an operad $\mathcal{P}=\{\mathcal{P}(n)\}$ as a dioperad via $\mathcal{P}(1, n):=\mathcal{P}(n)$ and $\mathcal{P}(m, n):=0$ for $m>1$.

(2) It may be of interests to relate dioperads to cyclic operads [GeK] or pseudotensor categories $[\mathrm{BD}]$, but the author does not know how to do this.

1.2. Let $V$ be an object of $\mathcal{C}$. Then the endomorphism dioperad $\mathcal{E n d}_{V}$ is defined by

$$
\operatorname{End}_{V}(m, n)=\mathcal{H} \operatorname{com}\left(V^{\otimes n}, V^{\otimes m}\right) .
$$

If $f \in \mathcal{E} n d_{V}\left(m_{1}, n_{1}\right), g \in \mathcal{E} n d_{V}\left(m_{2}, n_{2}\right)$, then $f_{i} \circ_{j} g \in \mathcal{E} n d_{V}\left(m_{1}+m_{2}-1, n_{1}+\right.$ $\left.n_{2}-1\right)$ is the morphism

$$
\begin{aligned}
&\left.(\mathrm{Id} \otimes \cdots \otimes f \otimes \cdots \otimes I d) \sigma(\operatorname{Id} \otimes \cdots \otimes g \otimes \cdots \otimes I d): V^{\otimes(} n_{1}+n_{2}-1\right) \\
& \rightarrow V^{\otimes\left(m_{1}+m_{2}-1\right)},
\end{aligned}
$$

where $f$ is at the $j$-th place, $g$ is at the $i$-th place, and $\sigma \in \mathbb{S}_{n_{1}+m_{2}-1}$ is the block permutation $((12)(45))_{i-1, j-1,1, m_{2}-j, n_{1}-i}$.

A morphism $f: \mathcal{P} \rightarrow \mathcal{Q}$ of dioperads in $\mathcal{C}$ is a collection of morphisms $f(m, n)$ : $\mathcal{P}(m, n) \rightarrow \mathcal{Q}(m, n), m, n \geq 1$, compatible with the structures of dioperads. If $\mathcal{P}$ is a dioperad in $\mathcal{C}$ and $V$ is an object of $\mathcal{C}$ equipped with a morphism $\mathcal{P} \rightarrow \mathcal{E} n d_{V}$, then $V$ is called a $\mathcal{P}$-algebra.

1.3. If $\mathcal{P}$ is a dioperad, then its opposite $\mathcal{P}^{\circ p}(m, n):=\mathcal{P}(n, m)$ with the transposed actions is a dioperad via the composition rule

$$
\stackrel{\stackrel{\mathrm{op}}{\circ}}{i}:=\left({ }_{j} \circ_{i}\right) \tau: \mathcal{P}^{\mathrm{op}}\left(m_{1}, n_{1}\right) \otimes \mathcal{P}^{\mathrm{op}}\left(m_{2}, n_{2}\right) \rightarrow \mathcal{P}^{\mathrm{op}}\left(m_{1}+m_{2}-1, n_{1}+n_{2}-1\right),
$$

where

$$
\tau: \mathcal{P}\left(n_{1}, m_{1}\right) \otimes \mathcal{P}\left(n_{2}, m_{2}\right) \stackrel{\sim}{\rightarrow} \mathcal{P}\left(n_{2}, m_{2}\right) \otimes \mathcal{P}\left(n_{1}, m_{1}\right)
$$

is the symmetry isomorphism.

If $\mathcal{P}$ and $\mathcal{Q}$ are dioperads, then $(\mathcal{P} \otimes \mathcal{Q})(m, n):=\mathcal{P}(m, n) \otimes \mathcal{Q}(m, n)$ with the diagonal actions of $\mathbb{S}_{m}$ and $\mathbb{S}_{n}$ is a dioperad via the composition rule

$$
\begin{aligned}
{\stackrel{\mathcal{P}}{\mathcal{P}} \stackrel{\mathcal{Q}}{\mathcal{Q}}_{j}:=\left({ }_{i} \circ_{j} \otimes_{i} \stackrel{\mathcal{O}}{\circ}_{j}\right.}^{\mathcal{P}}(\mathrm{Id} \otimes \tau \otimes \mathrm{Id}): & (\mathcal{P} \otimes \mathcal{Q})\left(m_{1}, n_{1}\right) \otimes(\mathcal{P} \otimes \mathcal{Q})\left(m_{2}, n_{2}\right) \\
& \rightarrow(\mathcal{P} \otimes \mathcal{Q})\left(m_{1}+m_{2}-1, n_{1}+n_{2}-1\right),
\end{aligned}
$$

where

$$
\tau: \mathcal{Q}\left(m_{1}, n_{1}\right) \otimes \mathcal{P}\left(m_{2}, n_{2}\right) \stackrel{\sim}{\rightarrow} \mathcal{P}\left(m_{2}, n_{2}\right) \otimes \mathcal{Q}\left(m_{1}, n_{1}\right)
$$

is the symmetry isomorphism. 
1.4. The suspension dioperad $\Sigma$ is the endomorphism dioperad of $\mathbb{k}[1]$, and the desuspension dioperad $\Sigma^{-1}$ is the endomorphism dioperad of $\mathbb{k}[-1]$; see eg. [Sm] $\S 3.1$. Thus, $\Sigma(m, n)$ is a one dimensional vector space placed in degree $n-m$ with sign representations of $\mathbb{S}_{m}$ and $\mathbb{S}_{n}$, and $\Sigma^{-1}(m, n)$ is a one dimensional vector space placed in degree $m-n$ with sign representations of $\mathbb{S}_{m}$ and $\mathbb{S}_{n}$. The suspension of a dioperad $\mathcal{P}$ is $\Sigma \mathcal{P}:=\Sigma \otimes \mathcal{P}$, and the desuspension is $\Sigma^{-1} \mathcal{P}:=$ $\Sigma^{-1} \otimes \mathcal{P}$.

Observe that if $\mathcal{P}$ is a dioperad, then $\{\mathcal{P}(m, n)[2 m-2]\}$ is also a dioperad. Define the sheared suspension dioperad $\Lambda$ by $\Lambda(m, n):=\Sigma(m, n)[2-2 m]$, and the sheared desuspension dioperad $\Lambda^{-1}$ by $\Lambda^{-1}(m, n):=\Sigma^{-1}(m, n)[2 m-2]$. Thus, $\Lambda(m, n)$ is a one dimensional vector space placed in degree $m+n-2$ with sign representations of $\mathbb{S}_{m}$ and $\mathbb{S}_{n}$, and $\Lambda^{-1}(m, n)$ is a one dimensional vector space placed in degree $2-m-n$ with sign representations of $\mathbb{S}_{m}$ and $\mathbb{S}_{n}$. The sheared suspension of a dioperad $\mathcal{P}$ is $\Lambda \mathcal{P}:=\Lambda \otimes \mathcal{P}$, and the sheared desuspension is $\Lambda^{-1} \mathcal{P}:=\Lambda^{-1} \otimes \mathcal{P}$.

\section{Quadratic dual}

From now on, for all dioperads $\mathcal{P}$, assume $\mathcal{P}(1,1)=\mathbb{k}$ unless otherwise stated.

2.1. By a tree, we shall always mean a directed tree such that each vertex has at least one outgoing edge and at least one incoming edge. A forest is a disjoint union of trees. If each vertex of a forest has valency at least three, then the forest is said to be reduced. Denote by Vert(T) (resp. edge(T), Edge(T)) the set of vertices (resp. internal edges, all edges) of a forest $T$, and Out(v) (resp. $\operatorname{In}(\mathrm{v})$ ) the set of outgoing (resp. incoming) edges at a vertex $v$. Let $\operatorname{det}(T):=\bigwedge^{\operatorname{ledge}(\mathrm{T}) \mid} \mathbb{k}^{\text {edge(T) }}$ and $\operatorname{Det}(\mathrm{T}):=\bigwedge^{|\operatorname{Edge}(\mathrm{T})|} \mathbb{k}^{\operatorname{Edge}(\mathrm{T})}$.

A $(m, n)$-tree is defined to be a tree with leaves labelled by $\{1, \ldots, n\}$ and roots labelled by $\{1, \ldots, m\}$. For any $(m, n)$-tree $T$, we have the formula

$$
\sum_{v \in \operatorname{Vert}(\mathrm{T})}(|\operatorname{Out}(\mathrm{v})|+|\operatorname{In}(\mathrm{v})|-2)=\mathrm{m}+\mathrm{n}-2 .
$$

Observe that the maximal number of vertices in a reduced $(m, n)$-tree is $m+n-2$. Note also that a trivalent $(m, n)$-tree has $m-1$ vertices with two outgoing edges and $n-1$ vertices with two incoming edges.

If $\underline{m}=\left(m_{1}, \ldots, m_{k}\right)$, where $m_{1}, \ldots, m_{k}$ are positive integers, then let $|\underline{m}|:=$ $m_{1}+\cdots+m_{k}$. Suppose $\underline{m}=\left(m_{1}, \ldots, m_{k}\right)$ and $\underline{n}=\left(n_{1}, \ldots, n_{k}\right)$. A $(\underline{m}, \underline{n})$ forest is defined to be a disjoint union of trees $T_{1}, \ldots, T_{k}$ such that, for each $i$, the leaves of $T_{i}$ are labelled by

$$
\left\{n_{1}+\cdots+n_{i-1}+1, \ldots, n_{1}+\cdots+n_{i-1}+n_{i}\right\}
$$

and the roots of $T_{i}$ are labelled by

$$
\left\{m_{1}+\cdots+m_{i-1}+1, \ldots, m_{1}+\cdots+m_{i-1}+m_{i}\right\} .
$$


2.2. Let $E(m, n), m, n \geq 1$, be a collection of finite dimensional $\left(\mathbb{S}_{m}, \mathbb{S}_{n}\right)$ bimodules with $E(1,1)=0$. If $T$ is a tree, let

$$
E(T)=\bigotimes_{v \in \operatorname{Vert}(\mathrm{T})} E(\operatorname{Out}(\mathrm{v}), \operatorname{In}(\mathrm{v}))
$$

The free dioperad $\mathcal{F}(E)$ generated by $E$ is defined by

$$
\mathcal{F}(E)(m, n):=\bigoplus_{(m, n)-\text { trees } T} E(T) .
$$

Let $(\Lambda E)(m, n):=\Lambda(m, n) \otimes E(m, n)$ and $\left(\Lambda^{-1} E\right)(m, n):=\Lambda^{-1}(m, n) \otimes$ $E(m, n)$. Observe that the canonical injections $(\Lambda E)(m, n) \hookrightarrow(\Lambda \mathcal{F}(E))(m, n)$ and $\left(\Lambda^{-1} E\right)(m, n) \hookrightarrow\left(\Lambda^{-1} \mathcal{F}(E)\right)(m, n)$ induce canonical dioperad isomorphisms

$$
\mathcal{F}(\Lambda E) \stackrel{\sim}{\rightarrow} \Lambda \mathcal{F}(E) \quad \text { and } \quad \mathcal{F}\left(\Lambda^{-1} E\right) \stackrel{\sim}{\rightarrow} \Lambda^{-1} \mathcal{F}(E)
$$

respectively.

2.3. Let $\mathcal{P}$ be a dioperad. An ideal $\mathcal{J}$ in $\mathcal{P}$ is a collection of $\left(\mathbb{S}_{m}, \mathbb{S}_{n}\right)$-subbimodules $\mathcal{J}(m, n) \subset \mathcal{P}(m, n)$ such that $f_{i} \circ_{j} g \in \mathcal{J}\left(m_{1}+m_{2}-1, n_{1}+n_{2}-1\right)$ for all $1 \leq i \leq n_{1}, 1 \leq j \leq m_{2}$, whenever $f \in \mathcal{J}\left(m_{1}, n_{1}\right), g \in \mathcal{P}\left(m_{2}, n_{2}\right)$, or $f \in \mathcal{P}\left(m_{1}, n_{1}\right), g \in \mathcal{J}\left(m_{2}, n_{2}\right)$. Intersection of ideals is an ideal. If $\mathcal{J}$ is an ideal in $\mathcal{P}$, then $(\mathcal{P} / \mathcal{J})(m, n):=\mathcal{P}(m, n) / \mathcal{J}(m, n)$ is a dioperad. The augmentation ideal of $\mathcal{P}$ is defined by $\overline{\mathcal{P}}(1,1):=0$ and $\overline{\mathcal{P}}(m, n):=\mathcal{P}(m, n)$ if $m+n \geq 3$.

2.4. Let $E(1,2)$ be a right $\mathbb{S}_{2}$-module, $E(2,1)$ be a left $\mathbb{S}_{2}$-module, and $E(m, n)=0$ for $(m, n) \neq(1,2),(2,1)$. Observe that

$$
\begin{gathered}
\mathcal{F}(E)(1,3)=\operatorname{Ind}_{\mathbb{S}_{2}}^{\mathbb{S}_{3}}(E(1,2) \otimes E(1,2)), \\
\mathcal{F}(E)(3,1)=\operatorname{Ind}_{\mathbb{S}_{2}}^{\mathbb{S}_{3}}(E(2,1) \otimes E(2,1)), \\
\mathcal{F}(E)(2,2)=(E(2,1) \otimes E(1,2)) \oplus \operatorname{Ind}_{\{1\}}^{\mathbb{S}_{2} \times \mathbb{S}_{2}}(E(1,2) \otimes E(2,1)) .
\end{gathered}
$$

Let $(R)$ be the ideal in $\mathcal{F}(E)$ generated by a right $\mathbb{S}_{3}$-submodule $R(1,3) \subset$ $\mathcal{F}(E)(1,3)$, a left $\mathbb{S}_{3}$-submodule $R(3,1) \subset \mathcal{F}(E)(3,1)$, and a $\left(\mathbb{S}_{2}, \mathbb{S}_{2}\right)$-subbimodule $R(2,2) \subset \mathcal{F}(E)(2,2)$. Denote by $\langle E ; R\rangle$ the dioperad $\mathcal{F}(E) /(R)$. A dioperad of the form $\langle E ; R\rangle$ is called a quadratic dioperad with generators $E$ and relations $R$. If $\mathcal{P}$ is a quadratic dioperad, then $\mathcal{P}(1,1)=\mathbb{k}$ and $\mathcal{P}=\langle E ; R\rangle$ where

$$
\begin{gathered}
E(i, j)=\mathcal{P}(i, j) \quad \text { for }(i, j)=(1,2),(2,1), \\
R(i, j)=\operatorname{Ker}(\mathcal{F}(E)(i, j) \rightarrow \mathcal{P}(i, j)) \quad \text { for }(i, j)=(1,3),(3,1),(2,2) .
\end{gathered}
$$


2.5. Let $\operatorname{Sgn}_{n}$ denote the sign representation of $\mathbb{S}_{n}$. If $V$ is a $\left(\mathbb{S}_{m}, \mathbb{S}_{n}\right)$-bimodule, then let $V^{*}:=\mathcal{H} \operatorname{Hom}(V, \mathbb{k})$ be the $\left(\mathbb{S}_{m}, \mathbb{S}_{n}\right)$-bimodule with the transposed actions, and let $V^{\vee}:=V^{*} \otimes\left(\operatorname{Sgn}_{\mathrm{m}} \otimes \operatorname{Sgn}_{\mathrm{n}}\right)$. If $\mathcal{P}=\langle E ; R\rangle$ is a quadratic dioperad, then its quadratic dual is defined by $\mathcal{P}^{!}:=\left\langle E^{\vee} ; R^{\perp}\right\rangle$, where $E^{\vee}(i, j):=E(i, j)^{\vee}$ for $(i, j)=(1,2),(2,1)$, and $R^{\perp}(i, j) \subset \mathcal{F}\left(E^{\vee}\right)(i, j)=\mathcal{F}(E)(i, j)^{\vee}$ is the orthogonal complement of $R(i, j)$ for $(i, j)=(1,3),(3,1),(2,2)$. Here, the identification $\mathcal{F}\left(E^{\vee}\right)=\mathcal{F}(E)^{\vee}$ is canonically defined as follows:

$$
\mathcal{F}\left(E^{\vee}\right)=\mathcal{F}\left(\Lambda^{-1} E^{*}[-1]\right) \stackrel{(2.2)}{\sim} \Lambda^{-1} \mathcal{F}\left(E^{*}[-1]\right)=\mathcal{F}(E)^{\vee},
$$

where the last equality follows from $[\mathrm{GiK}]$ Lemma 3.2.9(b).

Example. The quadratic dioperad $\mathcal{B} i$ Lie has generators $E(1,2)=\mathbb{k} \cdot l$, $E(2,1)=\mathbb{k} \cdot \delta$, both with sign actions of $\mathbb{S}_{2}$. The relations are spanned by

$$
\begin{gathered}
\left(l_{1} \circ_{1} l\right)+\left(l_{1} \circ_{1} l\right) \sigma+\left(l_{1} \circ_{1} l\right) \sigma^{2}, \\
\left(\delta_{1} \circ_{1} \delta\right)+\sigma\left(\delta_{1} \circ_{1} \delta\right)+\sigma^{2}\left(\delta_{1} \circ_{1} \delta\right), \\
\left(\delta_{1} \circ_{1} l\right)-\left(l_{1} \circ_{1} \delta\right)-\left(l_{1} \circ_{2} \delta\right)-\left(l_{2} \circ_{1} \delta\right)-\left(l_{2} \circ_{2} \delta\right),
\end{gathered}
$$

where $\sigma$ denotes the permutation $(123) \in \mathbb{S}_{3}$. Thus, $\mathcal{B} i \mathcal{L}$ ie-algebras are same as Lie bialgebras $[\mathrm{Dr}]$.

The quadratic dual $\mathcal{B} i \mathcal{L} i e^{!}$has generators $E(1,2)=\mathbb{k} \cdot m, E(2,1)=\mathbb{k} \cdot \Delta$, both with trivial actions of $\mathbb{S}_{2}$. The relations are spanned by

$$
\begin{array}{cl}
\left(m_{1} \circ_{1} m\right)-\left(m_{1} \circ_{1} m\right) \sigma, & \left(m_{1} \circ_{1} m\right)-\left(m_{1} \circ_{1} m\right) \sigma^{2}, \\
\left(\Delta_{1} \circ_{1} \Delta\right)-\sigma\left(\Delta_{1} \circ_{1} \Delta\right), & \left(\Delta_{1} \circ_{1} \Delta\right)-\sigma^{2}\left(\Delta_{1} \circ_{1} \Delta\right), \\
\left(\Delta_{1} \circ_{1} m\right)-\left(m_{1} \circ_{1} \Delta\right), & \left(\Delta_{1} \circ_{1} m\right)-\left(m_{1} \circ_{2} \Delta\right), \\
\left(\Delta_{1} \circ_{1} m\right)-\left(m_{2} \circ_{1} \Delta\right), & \left(\Delta_{1} \circ_{1} m\right)-\left(m_{2} \circ_{2} \Delta\right) .
\end{array}
$$

Thus, a $\mathcal{B} i$ L $i e^{!}$-algebra is a commutative algebra $A$ equipped with a cocommutative comultiplication $\Delta: A \rightarrow A \otimes A$ such that $\Delta$ is a map of $A$-modules. Abrams ([Ab] Theorem 1) has proved that unital, counital $\mathcal{B} i \mathcal{L} i e^{!}$-algebras are same as unital commutative Frobenius algebras. Observe that $\operatorname{BiL} i e^{!}(m, n)=\mathbb{k}$ for all $m, n$ (cf. $§ 5.2$ below).

\section{Remarks.}

(1) Infinitesimal bialgebras (cf. eg. $[\mathrm{Ag}]$ ) are also algebras over a quadratic dioperad.

(2) A dioperad $\mathcal{P}$ gives a PROP $\mathcal{Q}$ defined using the same generators and relations. Algebras over the dioperad $\mathcal{P}$ are same as algebras over the PROP Q. Since the relations defining $\mathcal{P}$ are expressed using trees, the grading of a free PROP by genus of graphs induces a grading on 2 . The bialgebra PROP does not have this grading because the compatibility relation between the multiplication and the comultiplication is expressed using a tree 
and a graph of genus 1 (cf. [ES]), hence bialgebras cannot be described by a dioperad. Thus, not all PROP's come from dioperads.

\section{Cobar dual}

3.1. Let $\mathcal{P}$ be a dioperad. The bicomplex $\mathcal{F}\left(\overline{\mathcal{P}}^{*}[-1]\right)(m, n)$ has differential $d^{\prime}+d^{\prime \prime}$, where $d^{\prime}$ is induced by the differential of $\mathcal{P}$ and $d^{\prime \prime}$ is induced by edge contractions $([\mathrm{GiK}](3.2 .3))$ :

$$
\overline{\mathcal{P}}(m, n)^{*} \stackrel{d^{\prime \prime}}{\rightarrow} \bigoplus_{|\operatorname{edge}(\mathrm{T})|=1} \overline{\mathcal{P}}^{*}(T) \otimes \operatorname{det}(T) \stackrel{d^{\prime \prime}}{\rightarrow} \bigoplus_{|\operatorname{edge}(\mathrm{T})|=2} \overline{\mathcal{P}}^{*}(T) \otimes \operatorname{det}(T) \stackrel{d^{\prime \prime}}{\rightarrow} \ldots
$$

where the sums are taken over reduced $(m, n)$-trees. In $(3.1)$, the leftmost term is placed in degree 1. The cobar complex $\mathbf{C P}(m, n)$ is defined to be the total complex of $\mathcal{F}\left(\overline{\mathcal{P}}^{*}[-1]\right)(m, n)$.

Define the cobar dual $\mathbf{D} \mathcal{P}$ of $\mathcal{P}$ by $\mathbf{D} \mathcal{P}:=\Lambda^{-1} \mathbf{C} \mathcal{P}$. Thus, $\mathbf{D} \mathcal{P}$ is the total complex of

$$
\overline{\mathcal{P}}(m, n)^{\vee} \stackrel{d^{\prime \prime}}{\rightarrow} \bigoplus_{|\operatorname{edge}(\mathrm{T})|=1} \overline{\mathcal{P}}^{*}(T) \otimes \operatorname{Det}(\mathrm{T}) \stackrel{\mathrm{d}^{\prime \prime}}{\rightarrow} \bigoplus_{|\operatorname{edge}(\mathrm{T})|=2} \overline{\mathcal{P}}^{*}(\mathrm{~T}) \otimes \operatorname{Det}(\mathrm{T}) \stackrel{\mathrm{d}^{\prime \prime}}{\rightarrow} \ldots
$$

where the sums are taken over reduced $(m, n)$-trees. In $(3.2)$, the leftmost term is placed in degree $3-m-n$. Note that $\mathbf{D} \mathcal{P}$ is also the total complex of the free dioperad generated by $\Lambda^{-1} \bar{P}^{*}[-1]$ with differential $d^{\prime}+d^{\prime \prime}$.

Proposition 3.3. There is a canonical quasi-isomorphism $\mathbf{D D} \mathcal{P} \rightarrow \mathcal{P}$.

Proof. (See [GiK] Theorem (3.2.16) or [MSS] Theorem 3.24.)

$$
\begin{aligned}
& \mathbf{D} \mathcal{P}(m, n)=\bigoplus_{(m, n) \text {-trees } T} \bigotimes_{v \in \operatorname{Vert}(\mathrm{T})} \overline{\mathcal{P}}(\operatorname{Out}(\mathrm{v}), \operatorname{In}(\mathrm{v}))^{*} \otimes \operatorname{Det}(\mathrm{T}) . \\
& \operatorname{DDP}(m, n)=\bigoplus_{(m, n) \text {-trees } T^{\prime}} \bigotimes_{w \in \operatorname{Vert}\left(\mathrm{T}^{\prime}\right)}\left[\bigoplus_{(\operatorname{Out}(\mathrm{v}), \operatorname{In}(\mathrm{v})) \text {-trees } T_{w}}\right. \\
& \left.\bigotimes_{v \in \operatorname{Vert}\left(\mathrm{T}_{\mathrm{w}}\right)} \overline{\mathcal{P}}(\operatorname{Out}(\mathrm{v}), \operatorname{In}(\mathrm{v}))^{*} \otimes \operatorname{Det}\left(\mathrm{T}_{\mathrm{w}}\right)\right]^{*} \otimes \operatorname{Det}\left(\mathrm{T}^{\prime}\right) \\
& =\bigoplus_{(m, n) \text {-trees } T^{\prime}(m, n) \text {-trees } T \geq T^{\prime}}\left(\bigotimes_{v \in \operatorname{Vert}(\mathrm{T})} \overline{\mathcal{P}}(\operatorname{Out}(\mathrm{v}), \operatorname{In}(\mathrm{v}))\right) \\
& \otimes\left(\bigotimes_{w \in \operatorname{Vert}\left(\mathrm{T}^{\prime}\right)} \operatorname{Det}\left(\mathrm{T}_{\mathrm{w}}\right)^{*}\right) \otimes \operatorname{Det}\left(\mathrm{T}^{\prime}\right) \\
& =\bigoplus_{(m, n) \text {-trees } T} \overline{\mathcal{P}}(T) \otimes\left(\bigoplus_{T^{\prime} \leq T} \bigotimes_{w \in \operatorname{Vert}\left(\mathrm{T}^{\prime}\right)} \operatorname{Det}\left(\mathrm{T}_{\mathrm{w}}\right)^{*} \otimes \operatorname{Det}\left(\mathrm{T}^{\prime}\right)\right),
\end{aligned}
$$


where $T_{w}$ is the subtree of $T$ contracted into $w$. Note that the summand corresponding to $T$ with just one vertex is $\mathcal{P}(m, n)$. From here on, continue as in [GiK] p.247.

3.2. Let $\mathcal{P}=\langle E ; R\rangle$ be a quadratic dioperad concentrated in degree 0 . Then

$$
\mathbf{D} \mathcal{P}(m, n)^{0}=\mathcal{F}\left(E^{\vee}\right)(m, n), \quad H^{0} \mathbf{D} \mathcal{P}(m, n)=\mathcal{P}^{!}(m, n) .
$$

If the canonical map $\mathbf{D} \mathcal{P}(m, n) \rightarrow \mathcal{P}^{!}(m, n)$ is a quasi-isomorphism for all $m, n \geq$ 1 , then $\mathcal{P}$ is said to be Koszul. If $\mathcal{P}$ is Koszul, then $\mathbf{D} \mathcal{P}$ !-algebras are called strong homotopy $\mathcal{P}$-algebras.

\section{Categorical cobar complex}

From now on, $\mathcal{P}$ denotes a dioperad concentrated in degree 0 unless otherwise stated.

4.1. Let $T$ be a forest as in $\S 2$ (not necessarily reduced). The direction on edges induces a partial ordering $\preceq$ on $\operatorname{Vert}(\mathrm{T})$. A surjective map $\ell$ : Vert $(\mathrm{T}) \rightarrow$ $\{1, \ldots, \mathrm{N}\}$ is called a $N$-level function on $T$ if $v \supsetneqq w$ implies $\ell(v) \lesseqgtr \ell(w)$. A $N$-level forest $(T, \ell)$ is a forest $T$ equipped with a $N$-level function $\ell$. A $N$-level forest $(T, \ell)$ is said to be $i$-saturated if each path from a leave to a root traverse some vertex $v$ such that $\ell(v)=i$ ( $v$ need not be the same for all paths). If $(T, \ell)$ is $i$-saturated for all $i$, then we say that it is saturated.

4.2. A $\mathbb{S}$-bimodule $\mathcal{P}=\{\mathcal{P}(m, n)\}$ is a collection consisting of a $\left(\mathbb{S}_{m}, \mathbb{S}_{n}\right)$ bimodule $\mathcal{P}(m, n)$ for each $m, n \geq 1$. We can consider $\mathbb{k}$ as a $\mathbb{S}$-bimodule via

$$
\mathbb{k}(m, n):= \begin{cases}\mathbb{k} & \text { if }(m, n)=(1,1), \\ 0 & \text { else. }\end{cases}
$$

Let $\mathcal{M}$ be the category of $\mathbb{S}$-bimodules $\mathcal{P}$ with $\mathcal{P}(1,1)$ nonzero. Define a monoidal structure on $\mathcal{M}$, with unit object $\mathbb{k}$, as follows: if $\mathcal{P}_{1}, \mathcal{P}_{2}$ are objects of $\mathcal{M}$, then let

$$
\left(\mathcal{P}_{1} \square \mathcal{P}_{2}\right)(m, n):=\bigoplus_{(T, \ell)} \bigotimes_{i=1}^{2} \bigotimes_{v \in \ell^{-1}(i)} \mathcal{P}_{i}(\operatorname{Out}(\mathrm{v}), \operatorname{In}(\mathrm{v})),
$$

where the direct sum is taken over all saturated 2-level $(m, n)$-trees $(T, \ell)$. Thus, if $\mathcal{P}_{1}, \ldots, \mathcal{P}_{N}$ are objects of $\mathcal{M}$, then

$$
\left(\mathcal{P}_{1} \square \ldots \square \mathcal{P}_{N}\right)(m, n)=\bigoplus_{(T, \ell)} \bigotimes_{i=1}^{N} \bigotimes_{v \in \ell^{-1}(i)} \mathcal{P}_{i}(\operatorname{Out}(\mathrm{v}), \operatorname{In}(\mathrm{v}))
$$

where the direct sum is taken over all saturated $N$-level $(m, n)$-trees $(T, \ell)$. Dioperads are precisely monoids in $\mathcal{M}$.

More generally, suppose $\mathcal{P}_{1}, \ldots, \mathcal{P}_{N}$ are $\mathbb{S}$-bimodules such that $\mathcal{P}_{i} \in \mathcal{M}$ for all $i \leq r$ and $\mathcal{P}_{i} \notin \mathcal{M}$ for all $i>r$. Let $\underline{m}=\left(m_{1}, \ldots, m_{k}\right)$ and $\underline{n}=\left(n_{1}, \ldots, n_{k}\right)$. Then we define $\left(\mathcal{P}_{1} \square \ldots \square \mathcal{P}_{N}\right)(\underline{m}, \underline{n})$ by the right-hand side of $(4.1)$, but with 
the direct sum taken over all $N$-level $(\underline{m}, \underline{n})$-forests $(T, \ell)$ such that $(T, \ell)$ is $i$-saturated for all $i \leq r$.

4.3. Let $\mathcal{P}$ be a dioperad. Then the categorical cobar complex $\mathbf{L} \mathcal{P}$ of $\mathcal{P}$ is

$$
\overline{\mathcal{P}}^{*} \rightarrow \overline{\mathcal{P}}^{* \square 2} \rightarrow \overline{\mathcal{P}}^{* \square 3} \rightarrow \ldots,
$$

where the leftmost term is placed in degree 1 ; see $[\mathrm{MSS}] \S 3.4$ or [SVO] p.398. More generally, let $\mathbf{L} \mathcal{P}(\underline{m}, \underline{n})$ be the complex

$$
\overline{\mathcal{P}}^{*}(\underline{m}, \underline{n}) \rightarrow \overline{\mathcal{P}}^{* \square 2}(\underline{m}, \underline{n}) \rightarrow \overline{\mathcal{P}}^{* \square 3}(\underline{m}, \underline{n}) \rightarrow \ldots,
$$

where the leftmost term is placed in degree 1.

Proposition 4.2. If $m_{i}+n_{i} \geq 3$ for all $i$, then

$$
H(\mathbf{L} \mathcal{P}(\underline{m}, \underline{n}))=H\left(\mathbf{C P}\left(m_{1}, n_{1}\right)\right) \otimes \ldots \otimes H\left(\mathbf{C P}\left(m_{k}, n_{k}\right)\right) .
$$

In particular, if $m+n \geq 3$, then $H(\mathbf{L} \mathcal{P}(m, n))=H(\mathbf{C P}(m, n))$.

Define a decreasing filtration on $\mathbf{L} \mathcal{P}(\underline{m}, \underline{n})$ by letting $F^{p} \mathbf{L} \mathcal{P}(\underline{m}, \underline{n})$ be the subcomplex spanned by forests with at least $p$ vertices. This gives a spectral sequence

$$
E_{0}^{p, q}=\frac{F^{p} \mathbf{L} \mathcal{P}(\underline{m}, \underline{n})^{p+q}}{F^{p+1} \mathbf{L} \mathcal{P}(\underline{m}, \underline{n})^{p+q}} \Longrightarrow H^{p+q}(\mathbf{L} \mathcal{P}(\underline{m}, \underline{n})) .
$$

Hence, Proposition 4.2 follows from the following lemma.

Lemma 4.4. If $m_{i}+n_{i} \geq 3$ for all $i$, then

$$
E_{1}^{\bullet, 0} \cong \mathbf{C P}\left(m_{1}, n_{1}\right) \otimes \ldots \otimes \mathbf{C P}\left(m_{k}, n_{k}\right),
$$

and $E_{1}^{p, q}=0$ if $q \neq 0$.

The rest of this section is devoted to the proof of Lemma 4.4, which is same as the proofs in [MSS] $\S 3.6$ and [SVO] $\S 7$ except for the following modification: instead of using their surjection algebra, use another algebra that we next define.

4.4. Let $X$ be a finite set, and let $S=\left\{X_{i}\right\}_{i \in I}$ be a collection of nonempty subsets $X_{i}$ of $X$ such that:

(a) $X=\bigcup X_{i}$;

(b) if $\emptyset \neq Y \subset X_{i}$ for some $i$, then $Y \in S$;

(c) if $\emptyset \neq Y \subset X$ and all subsets of $Y$ with 2 elements are in $S$, then $Y \in S$.

Define an associative graded $\mathbb{k}$-algebra $A$ as follows: as a vector space, $A$ has a basis $\{1\} \cup\left\{a_{i}\right\}_{i \in I}$; multiplication of basis elements is defined by

$$
a_{i} a_{j}:= \begin{cases}a_{k} & \text { if } X_{i} \cap X_{j}=\emptyset \& X_{i} \cup X_{j}=X_{k}, \\ 0 & \text { else. }\end{cases}
$$

The degree of $a_{i}$ is the number of elements of $X_{i}$. Equivalently, $A$ is a quadratic algebra with generators $a_{x}$ indexed by elements of $X$, and relations

$$
a_{x} a_{y}:= \begin{cases}a_{y} a_{x} & \text { if } x \neq y \&\{x, y\} \in S \\ 0 & \text { else }\end{cases}
$$


Thus, $a_{i}$ is the product of all $a_{x}$ with $x \in X_{i}$.

Lemma 4.5. The quadratic algebra $A$ is Koszul.

Proof. A PBW basis for $A$ is defined by choosing a simple order on the generators $a_{x}$, and then for each $i$, choose the admissible expression $a_{i}=a_{x} a_{y} \ldots a_{z}$ to be the one which is maximal in the lexicographical ordering; cf. [MSS] Proposition 3.66 or [SVO] Proposition 19. Hence, $A$ is Koszul by [Pr] Theorem 5.3.

4.5. Fix a forest $T$ such that each component of $T$ has at least one vertex. Call $V \subset \operatorname{Vert}(\mathrm{T})$ a level of $T$ if there exists a level function $\ell$ with $V=\ell^{-1}(i)$ for some $i$. Let $X=\operatorname{Vert}(\mathrm{T})$ and let $S$ be the collection of all levels of $T$. Then $X$ and $S$ satisfy the conditions in $\S 4.4$. (To verify condition (c), use induction on the number of elements in $Y$.) Thus, there is a corresponding algebra $A$. Recall that $A$ has a basis labelled by 1 and all the levels.

Call a sequence $V_{1}, \ldots, V_{N}$ of levels good if there exists a $N$-level function $\ell$ such that $V_{i}=\ell^{-1}(i)$ for all $i$. Consider the cochain complex $C^{\bullet}(A):=\bar{A}^{* \otimes \bullet}$. For each $N$, there is a direct sum decompositon $\bar{A}^{\otimes N}=U_{N} \oplus V_{N}$, where $U_{N}$ is spanned by $a_{i_{1}} \otimes \ldots \otimes a_{i_{N}}$ such that $X_{i_{1}}, \ldots, X_{i_{N}}$ is not a good sequence of levels, and $V_{N}$ is spanned by $a_{i_{1}} \otimes \ldots \otimes a_{i_{N}}$ such that $X_{i_{1}}, \ldots, X_{i_{N}}$ is a good sequence of levels. Let $C_{U}^{N}$ be the annihilator of $V_{N}$ and $C_{V}^{N}$ be the annihilator of $U_{N}$. Denote by $\partial$ the boundary map of the chain complex $\bar{A}^{\otimes \bullet}$. Since $\partial\left(U_{N}\right) \subset U_{N-1}$ and $\partial\left(V_{N}\right) \subset V_{N-1}$, it follows that $C^{\bullet}(A)=C_{U}^{\bullet} \oplus C_{V}^{\bullet}$, a direct sum of two subcomplexes.

Let $\left\{a_{i}^{*}\right\}$ be the dual basis of $\left\{a_{i}\right\}$, then $C_{V}^{N}$ has a basis consisting of all $a_{i_{1}}^{*} \otimes \ldots \otimes a_{i_{N}}^{*}$ for which $X_{i_{1}}, \ldots, X_{i_{N}}$ is a good sequence of levels. The differential on $C_{V}^{N}$ is

$$
\begin{gathered}
\delta\left(a_{i_{1}}^{*} \otimes \ldots \otimes a_{i_{N}}^{*}\right)=\sum(-1)^{r} a_{i_{1}}^{*} \otimes \ldots \otimes \delta\left(a_{i_{r}}^{*}\right) \otimes \ldots \otimes a_{i_{N}}^{*}, \\
\delta\left(a_{i}^{*}\right)=\sum_{X_{j} \amalg X_{k}=X_{i}} a_{j}^{*} \otimes a_{k}^{*}
\end{gathered}
$$

Lemma 4.6. If $N \neq|\operatorname{Vert}(\mathrm{T})|$, then $H^{N}\left(C_{V}^{\bullet}\right)=0$. If $N=|\operatorname{Vert}(\mathrm{T})|$, then $H^{N}\left(C_{V}^{\bullet}\right)=\operatorname{det}(T)$.

Proof. (See [MSS] Proposition 3.68 or [SVO] Proposition 21.)

Note that $H\left(C^{\bullet}(A)\right)=H\left(C_{U}^{\bullet} \oplus H\left(C_{V}^{\bullet}\right)\right.$. Let $\left[a_{i_{1}}^{*} \otimes \ldots \otimes a_{i_{N}}^{*}\right] \in H^{N}\left(C_{V}^{\bullet}\right)$ be a nonzero cohomology class. By Lemma $4.5, A$ is Koszul. Therefore, $X_{i_{1}}, \ldots, X_{i_{N}}$ must each be a one element subset of $\operatorname{Vert}(\mathrm{T})$. But $X_{i_{1}}, \ldots, X_{i_{N}}$ is a good sequence of levels, hence $N=|\operatorname{Vert}(\mathrm{T})|$.

Define a map $\iota: C_{V}^{N} \rightarrow \bigwedge^{|\operatorname{Vert}(\mathrm{T})|} \mathbb{k}^{\operatorname{Vert}(\mathrm{T})} \cong \operatorname{det}(T)$ by sending $a_{x}^{*} \otimes a_{y}^{*} \otimes \ldots \otimes a_{z}^{*}$ to $x \wedge y \wedge \ldots \wedge z$, where $\{x\},\{y\}, \ldots,\{z\}$ is any good sequence of levels. The coboundaries in $C_{V}^{N}$ are spanned by terms of the form $a_{x}^{*} \otimes \ldots \otimes \delta a_{\left\{y_{1}, y_{2}\right\}}^{*} \otimes \ldots \otimes a_{z}^{*}$ with

$$
\delta a_{\left\{y_{1}, y_{2}\right\}}^{*}=a_{y_{1}}^{*} \otimes a_{y_{2}}^{*}+a_{y_{2}}^{*} \otimes a_{y_{1}}^{*}
$$


hence $\iota$ induces a map $H^{N}\left(C_{V}^{\bullet}\right) \rightarrow \operatorname{det}(T)$. Clearly, $\iota$ is surjective. Observe that any two good sequences of singleton levels can be obtained from each other by performing transpositions of succesive levels while remaining good at the intermediate steps. This implies that $H^{N}\left(C_{V}^{\bullet}\right)$ is at most one dimensional. Hence $\iota$ is bijective.

4.6. Suppose $m_{i}+n_{i} \geq 3$ for all $i$. For a $(\underline{m}, \underline{n})$-forest $T$ with $p$ vertices, let

$$
E_{0}^{p, \bullet}(T):=\overline{\mathcal{P}}^{*}\left(T_{1}\right) \otimes \cdots \otimes \overline{\mathcal{P}}^{*}\left(T_{k}\right) \otimes C_{V}^{p+\bullet},
$$

where $T_{1}, \ldots, T_{k}$ are the connected components of $T$. Observe that the complex $E_{0}^{p, \bullet}$ of (4.3) is the direct sum of $E_{0}^{p, \bullet}(T)$ over all $(\underline{m}, \underline{n})$-forests $T$ with $p$ vertices. Therefore, Lemma 4.6 implies Lemma 4.4.

\section{Koszul complex}

From now on, assume that $\mathcal{P}$ is quadratic unless otherwise stated.

5.1. Observe that a summand in $\mathcal{P} \square\left(\Lambda \mathcal{P}^{!}\right)^{*}$ corresponding to a saturated 2level tree $(T, \ell)$ is placed in degree $\sum_{v \in \ell^{-1}(2)}(2-|\operatorname{Out}(\mathrm{v})|-|\operatorname{In}(\mathrm{v})|)$. The Koszul complex of a quadratic dioperad $\mathcal{P}=\langle E ; R\rangle$ is defined to be $\mathbf{K} \mathcal{P}:=\mathcal{P} \square\left(\Lambda \mathcal{P}^{!}\right)^{*}$ with differential $d$ defined as follows.

Let

$$
\mu: \mathcal{P} \square \mathcal{P} \rightarrow \mathcal{P} \quad \text { and } \quad \delta:\left(\Lambda \mathcal{P}^{!}\right)^{*} \rightarrow\left(\Lambda \mathcal{P}^{!}\right)^{*} \square\left(\Lambda \mathcal{P}^{!}\right)^{*}
$$

be, respectively, the composition map of $\mathcal{P}$ and the dual of the composition map of $\Lambda P^{\text {! }}$. Let

$$
\alpha(1,2): E(1,2)[1] \rightarrow E(1,2), \quad \alpha(2,1): E(2,1)[1] \rightarrow E(2,1)
$$

be the identity maps of degree 1 . Then $d$ is defined to be the composition

$$
\mathcal{P} \square\left(\Lambda \mathcal{P}^{!}\right)^{*} \stackrel{\operatorname{Id} \square \delta}{\longrightarrow} \mathcal{P} \square\left(\Lambda \mathcal{P}^{!}\right)^{*} \square\left(\Lambda \mathcal{P}^{!}\right)^{*} \stackrel{\Phi}{\longrightarrow} \mathcal{P} \square \mathcal{P} \square\left(\Lambda \mathcal{P}^{!}\right)^{*} \stackrel{\mu \square \mathrm{Id}}{\longrightarrow} \mathcal{P} \square\left(\Lambda \mathcal{P}^{!}\right)^{*},
$$

where $\Phi$ is defined to be $\operatorname{Id} \square \alpha \square$ Id on saturated 3-level trees whose second level has one vertex of valency 3 and all other second level vertices are of valency 2 , and zero otherwise.

The proof of the next lemma is a standard argument for twisted tensor products.

Lemma 5.1. The map $d$ is a differential, i.e. $d d=0$.

Proof.

$$
\begin{aligned}
& d d=(\mu \square \operatorname{Id}) \Phi(\operatorname{Id} \square \delta)(\mu \square \operatorname{Id}) \Phi(\operatorname{Id} \square \delta) \\
& =(\mu \square \mathrm{Id}) \Phi(\mu \square \operatorname{Id} \square \operatorname{Id})(\operatorname{Id} \square \operatorname{Id} \square \delta) \Phi(\operatorname{Id} \square \delta) \\
& =(\mu \square \operatorname{Id})(\mu \square \operatorname{Id} \square \operatorname{Id})(\operatorname{Id} \square \Phi)(\Phi \square \operatorname{Id})(\operatorname{Id} \square \operatorname{Id} \square \delta)(\operatorname{Id} \square \delta) \\
& =(\mu \square \operatorname{Id})(\operatorname{Id} \square \mu \square \operatorname{Id})(\operatorname{Id} \square \Phi)(\Phi \square \operatorname{Id})(\operatorname{Id} \square \delta \square \operatorname{Id})(\operatorname{Id} \square \delta) .
\end{aligned}
$$

But $(\operatorname{Id} \square \mu \square \operatorname{Id})(\operatorname{Id} \square \Phi)(\Phi \square \operatorname{Id})(\operatorname{Id} \square \delta \square \mathrm{Id})=0$ by relations defining $\mathcal{P}$ (in the case when the second level has one vertex of valency 4 and all other second 
level vertices are of valency 2) and by Koszul sign rule (in the case when the second level has two vertices of valency 3 and all other second level vertices are of valency 2 ).

Theorem 5.2. A quadratic dioperad $\mathcal{P}$ is Koszul if and only if the canonical map $\mathbf{K P} \rightarrow \mathbb{k}$ is a quasi-isomorphism (i.e. $\mathbf{K P}(m, n)$ is exact for all $(m, n) \neq(1,1))$.

Proof. As usual, there is an exact sequence $\mathbf{B P}(m, n)$ :

$$
(\mathcal{P} \square \overline{\mathcal{P}} \square \cdots \square \overline{\mathcal{P}})(m, n) \rightarrow \cdots \rightarrow(\mathcal{P} \square \overline{\mathcal{P}})(m, n) \rightarrow \mathcal{P}(m, n) \rightarrow \mathbb{k}(m, n),
$$

where the leftmost term is placed in degree $1-m-n$ and the rightmost term is placed in degree 0 . Suppose $m+n \geq 3$. Define a decreasing filtration on $\mathbf{B P}(m, n)$ by letting $F^{p} \mathbf{B} \mathcal{P}(m, n)$ be the subcomplex spanned by 1-saturated $(m, n)$-trees $(T, \ell)$ with

$$
\sum_{v \in \ell^{-1}(1)}(|\operatorname{Out}(\mathrm{v})|+|\operatorname{In}(\mathrm{v})|-2) \geq \mathrm{p} .
$$

This gives a spectral sequence

$$
E_{0}^{p, q}=\frac{F^{p} \mathbf{B} \mathcal{P}(m, n)^{p+q}}{F^{p+1} \mathbf{B} \mathcal{P}(m, n)^{p+q}} \Longrightarrow H^{p+q}(\mathbf{B} \mathcal{P}(m, n))=0 .
$$

Let $(T, \ell)$ be a saturated 2-level $(m, n)$-tree. Suppose

$$
\left\{v_{1}, \ldots, v_{k}\right\}:=\left\{v \in \ell^{-1}(2) ;|\operatorname{Out}(\mathrm{v})|+|\operatorname{In}(\mathrm{v})| \geq 3\right\} \neq \emptyset .
$$

Let

$$
\underline{r}:=\left(\left|\operatorname{Out}\left(\mathrm{v}_{1}\right)\right|, \ldots,\left|\operatorname{Out}\left(\mathrm{v}_{\mathrm{k}}\right)\right|\right) \quad \text { and } \quad \underline{\mathrm{s}}:=\left(\left|\operatorname{In}\left(\mathrm{v}_{1}\right)\right|, \ldots,\left|\operatorname{In}\left(\mathrm{v}_{\mathrm{k}}\right)\right|\right) .
$$

Note that, by (2.1), we have

$$
|\underline{r}|+|\underline{s}|-2 k+\sum_{v \in \ell^{-1}(1)}(|\operatorname{Out}(\mathrm{v})|+|\operatorname{In}(\mathrm{v})|-2)=m+n-2 .
$$

Let

$$
E_{0}^{p, \bullet}(T, \ell):=\bigotimes_{v \in \ell^{-1}(1)} \mathcal{P}(\operatorname{Out}(\mathrm{v}), \operatorname{In}(\mathrm{v})) \otimes \mathbf{L} \mathcal{P}(\underline{\mathrm{r}}, \underline{\mathrm{s}})^{*}[\mathrm{p}+1] .
$$

Observe that, for $0 \leq p \leq m+n-3$, the complex $E_{0}^{p, \bullet}$ of (5.3) is the direct sum of $E_{0}^{p, \bullet}(T, \ell)$ over all saturated 2-level $(m, n)$-trees $(T, \ell)$ such that

$$
\sum_{v \in \ell^{-1}(1)}(|\operatorname{Out}(\mathrm{v})|+|\operatorname{In}(\mathrm{v})|-2)=\mathrm{p} .
$$

By (3.4) and Proposition 4.2, $H^{|\underline{r}|+|\underline{s}|-2 k}(\mathbf{L} \mathcal{P}(\underline{r}, \underline{s}))=\left(\Lambda \mathcal{P}^{!}\right)(\underline{r}, \underline{s})$. Hence, by $(5.4)-(5.6)$,

$$
E_{1}^{\bullet, 1-m-n}=\mathbf{K} \mathcal{P}(m, n)[2-m-n] .
$$

Now assume that $\mathcal{P}$ is Koszul. Suppose $(m, n) \neq(1,1)$. By Proposition 4.2 and (5.4)-(5.6), $E_{1}^{p, q}=0$ if $q \neq 1-m-n$. Hence, by (5.7), $\mathbf{K P}(m, n)$ is exact. 
Conversely, assume $\mathbf{K} \mathcal{P}(m, n)$ is exact for all $(m, n) \neq(1,1)$. Let $\underline{w}:=$ $\left(w_{1}, \ldots, w_{j}\right)$ and $\underline{x}:=\left(x_{1}, \ldots, x_{j}\right)$, where $w_{l}+x_{l} \geq 3$ for all $l$. We shall prove, by induction on $|\underline{w}|+|\underline{x}|-2 j$, that

$$
H^{i}(\mathbf{L} \mathcal{P}(\underline{w}, \underline{x}))=0 \quad \text { if } i<|\underline{w}|+|\underline{x}|-2 j .
$$

By Proposition 4.2, this would imply that $\mathcal{P}$ is Koszul.

First, note that (5.8) is true if $|\underline{w}|+|\underline{x}|-2 j=1$. Assume that (5.8) is true if $|\underline{w}|+|\underline{x}|-2 j<N$. Now let $|\underline{w}|+|\underline{x}|-2 j=N$. By Proposition 4.2, it suffices to show that, for each $l$, we have $H^{i}\left(\mathbf{L} \mathcal{P}\left(w_{l}, x_{l}\right)\right)=0$ if $i<w_{l}+x_{l}-2$. Consider the spectral sequence (5.3) with $m:=w_{l}$ and $n:=x_{l}$. Note that by $(5.5)-(5.6), E_{0}^{0, \bullet}=\mathbf{L} \mathcal{P}(m, n)^{*}[1]$, so $E_{1}^{0, q}=H^{q+1}\left(\mathbf{L} \mathcal{P}(m, n)^{*}\right)$. Moreover, by induction hypothesis and (5.4)-(5.6), we have $E_{1}^{p, q}=0$ if $p>0$ and $q>1-m-n$. Hence, by (5.7) and exactness of $\mathbf{K} \mathcal{P}(m, n)$, we obtain

$$
E_{2}^{p, q}= \begin{cases}H^{q+1}\left(\mathbf{L} \mathcal{P}(m, n)^{*}\right) & \text { if } p=0 \text { and } q \geq 2-m-n, \\ 0 & \text { else. }\end{cases}
$$

Therefore, $H^{i}(\mathbf{L} \mathcal{P}(m, n))=0$ for $i<m+n-2$.

5.2. Observe that if $\mathcal{P}$ is a quadratic dioperad, then $\mathcal{A}(n):=\mathcal{P}(1, n)$ and $\mathcal{B}(n):=\mathcal{P o p}(1, n)$ are quadratic operads. The inclusions $\mathcal{A} \hookrightarrow \mathcal{P}$ and $\mathcal{B}^{\circ \mathrm{p}} \hookrightarrow \mathcal{P}$ induce canonical morphisms $\mathcal{A} \square \mathcal{B}^{\mathrm{op}} \rightarrow \mathcal{P}$ and $\mathcal{B}^{\mathrm{op}} \square \mathcal{A} \rightarrow \mathcal{P}$. The following proposition is adapted from [M2]; cf. also [FM].

Proposition 5.9. Let $\mathcal{P}=\langle E ; R\rangle$ be a quadratic dioperad, let $\mathcal{A}(n):=\mathcal{P}(1, n)$, and let $\mathcal{B}(n):=\mathcal{P}^{\circ p}(1, n)$.

(a) If $\mathcal{P}(m, n)=\left(\mathcal{A} \square \mathcal{B}^{\circ \mathrm{p}}\right)(m, n)$ (resp. $\mathcal{P}(m, n)=\left(\mathcal{B}^{\circ \mathrm{p}} \square \mathcal{A}\right)(m, n)$ ) for $(m, n)=$ $(2,2),(2,3)$, and $(3,2)$, then $\mathcal{P}=\mathcal{A} \square \mathcal{B}^{\circ \mathrm{p}}$ (resp. $\left.\mathcal{P}=\mathcal{B}^{\circ \mathrm{p}} \square \mathcal{A}\right)$.

(b) We have $\mathcal{P}=\mathcal{A} \square \mathcal{B}^{\text {op }}$ if and only if $\mathcal{P}^{!}=\mathcal{B}^{\text {!op }} \square \mathcal{A}^{!}$.

(c) If $\mathcal{A}$ and $\mathcal{B}$ are Koszul, and $\mathcal{P}=\mathcal{A} \square \mathcal{B}^{\circ p}$, then $\mathcal{P}$ is Koszul.

Proof.

(a) (See $[\mathrm{M} 2]$ Theorem 2.3.)

Note that $\mathcal{F}(E)(2,2)=\left(\mathcal{A} \square \mathcal{B}^{\circ \mathrm{p}}\right)(2,2) \oplus\left(\mathcal{B}^{\mathrm{op}} \square \mathcal{A}\right)(2,2)$. We have $\mathcal{P}(2,2)=$ $\left(\mathcal{A} \square \mathcal{B}^{\circ \mathrm{p}}\right)(2,2)$ if and only if there is a $\left(\mathbb{S}_{2}, \mathbb{S}_{2}\right)$-equivariant map

$$
\lambda:\left(\mathcal{B}^{\mathrm{op}} \square \mathcal{A}\right)(2,2) \rightarrow\left(\mathcal{A} \square \mathcal{B}^{\mathrm{op}}\right)(2,2)
$$

such that

$$
R(2,2)=\left\{x-\lambda(x) ; x \in\left(\mathcal{B}^{\circ \mathrm{p}} \square \mathcal{A}\right)(2,2)\right\} .
$$

The rest of the proof is same as [M2] Theorem 2.3. Similarly in the other case.

(b) (See [M2] Lemma 4.3.)

Assume that $\mathcal{P}=\mathcal{A} \square \mathcal{B}^{\text {op }}$, and let $\lambda$ be the map in the proof of (a). Via the identification (2.3), we obtain

$$
R^{\perp}(2,2)=\left\{\alpha-\lambda^{\vee}(\alpha) ; \alpha \in\left(\mathcal{A}^{!} \square \mathcal{B}^{! o p}\right)(2,2)\right\},
$$


where $\lambda^{\vee}$ is the dual of $\lambda$. It follows by a direct verification, using (a), that $\mathcal{P}^{!}=\mathcal{B}^{\text {!op }} \square \mathcal{A}^{!}$. Similarly for the converse.

(c) (See [M2] Proposition 4.4 and Theorem 4.5.)

Observe that $\overline{\mathcal{P}} \square \overline{\mathcal{P}} \subset \mathcal{F}(\overline{\mathcal{P}})$. Thus, using (2.2), we have

$$
\mathbf{K P}=\mathcal{A} \square \mathcal{B}^{\mathrm{op}} \square\left(\Lambda\left(\mathcal{B}^{! \mathrm{op}} \square \mathcal{A}^{!}\right)\right)^{*}=\mathcal{A} \square \mathcal{B}^{\mathrm{op}} \square\left(\Lambda \mathcal{B}^{\mathrm{op} !}\right)^{*} \square\left(\Lambda \mathcal{A}^{!}\right)^{*} .
$$

Suppose $(m, n) \neq(1,1)$. Define a decreasing filtration on $\mathbf{K P}(m, n)$ by letting $F^{p} \mathbf{K P}(m, n)$ be the subcomplex spanned by saturated 4-level $(m, n)$ trees $(T, \ell)$ with

$$
\sum_{v \in \ell^{-1}(4)}(2-|\operatorname{Out}(\mathrm{v})|-|\operatorname{In}(\mathrm{v})|) \geq \mathrm{p} .
$$

This gives a spectral sequence

$$
E_{0}^{p, q}=\frac{F^{p} \mathbf{K} \mathcal{P}(m, n)^{p+q}}{F^{p+1} \mathbf{K} \mathcal{P}(m, n)^{p+q}} \Longrightarrow H^{p+q} \mathbf{K} \mathcal{P}(m, n) .
$$

Now $E_{0}^{p, \bullet}$ is the subcomplex of $\mathcal{A} \square \mathbf{K} \mathcal{B}^{\circ \mathrm{p}} \square\left(\Lambda \mathcal{A}^{!}\right)^{*}$ (with differential $\operatorname{Id} \square d \square \mathrm{Id})$ spanned by saturated 3-level $(m, n)$-trees $(T, \ell)$ with

$$
\sum_{v \in \ell^{-1}(3)}(2-|\operatorname{Out}(\mathrm{v})|-|\operatorname{In}(\mathrm{v})|)=\mathrm{p} .
$$

Thus, $E_{1}^{p, q}=0$ if $q \neq 0$ and $E_{1}^{\bullet, 0}=\mathbf{K} \mathcal{A}(m, n)$. Hence, $E_{2}^{p, q}=0$ for all $p, q$.

Corollary 5.10. The dioperad BiLie is Koszul.

Proof. This is immediate from Proposition 5.9 and Koszulity of the Lie operad Lie ([BG] (6.4) or [GiK] Corollary (4.2.7)). We have: BiLie $=\mathcal{L} i e \square \mathcal{L} i e^{\mathrm{op}}$, $\mathcal{B} i \mathcal{L} i e^{!}=\operatorname{Com}^{\circ \mathrm{p}} \square \operatorname{Com}$, where $\operatorname{Com}=\mathcal{L} i e^{!}$.

\section{Remarks.}

(1) Note that, in more explicit terms, the cobar dual of $\mathcal{B} i \mathcal{L} i e^{!}$is the complex of trees with multiple leaves and multiple roots. The Koszulity of $\mathcal{B} i \mathcal{L} i e^{\text {! }}$ means that the cohomology of its cobar dual is zero in all negative degrees, but the author does not know of a direct proof of this.

(2) Similarly, one can show that the infinitesimal bialgebra dioperad is Koszul.

\section{Acknowledgements}

I am very grateful to Victor Ginzburg for suggesting the problem and for many useful discussions. I also thank Martin Markl for answering questions on his paper [M2]. 


\section{References}

[Ab] L. Abrams, Two-dimensional topological quantum field theories and Frobenius algebras, J. Knot Theory Ramifications 5 (1996), 569-587.

[Ad] J. F. Adams, Infinite loop spaces, Annals of Mathematics Studies, 90. Princeton University Press, Princeton, NJ, 1978.

[Ag] M. Aguiar, On the associative analog of Lie bialgebras, J. Algebra 244 (2001), 492-532.

[BD] A. Beilinson, V. Drinfeld, Chiral algebras, preprint, 1995.

[BG] A. Beilinson, V. Ginzburg, Infinitesimal structure of moduli spaces of G-bundles, Internat. Math. Res. Notices 1992, 63-74.

[Ch] M. Chas, Combinatorial Lie bialgebras of curves on surfaces, preprint: math.GT/0105178

[ChS] M. Chas, D. Sullivan, String topology, preprint: math.GT/9911159

[Dr] V. Drinfeld, Hamiltonian structures on Lie groups, Lie bialgebras and the geometric meaning of classical Yang-Baxter equations, Soviet Math. Dokl. 27 (1983), 68-71.

[ES] P. Etingof, O. Schiffmann, Lectures on quantum groups, Lectures in Mathematical Physics, International Press, Boston, MA, 1998.

[FM] T. Fox, M. Markl, Distributive laws, bialgebras, and cohomology, Operads: Proceedings of Renaissance Conferences (Hartford, CT/Luminy, 1995), 167-205, Contemp. Math., 202, Amer. Math. Soc., Providence, RI, 1997.

[Ge] E. Getzler, Operads and moduli spaces of genus 0 Riemann surfaces, The moduli space of curves (Texel Island, 1994), 199-230, Progr. Math., 129, Birkhäuser Boston, Boston, MA, 1995.

[GeK] E. Getzler, M. Kapranov, Cyclic operads and cyclic homology, Geometry, topology, \& physics, 167-201, Conf. Proc. Lecture Notes Geom. Topology, IV, International Press, Cambridge, MA, 1995.

[GiK] V. Ginzburg, M. Kapranov, Koszul duality for operads, Duke Math. J. 76 (1994), 203-272.

[Ka] M. Kapranov, Operads and algebraic geometry, Proceedings of the International Congress of Mathematicians, Vol. II (Berlin, 1998). Doc. Math. 1998, Extra Vol. II, 277286.

[Ko] M. Kontsevich, Formal (non) commutative symplectic geometry, The Gelfand Mathematical Seminars, 1990-1992, 173-187, Birkhäuser Boston, Boston, MA, 1993.

[L] J.-L. Loday, La renaissance des opérades, Séminaire Bourbaki, Vol. 1994/95. Astérisque No. 237, (1996), Exp. No. 792, 3, 47-74.

[M1] M. Markl, Models for operads, Comm. Algebra 24 (1996), 1471-1500.

[M2] _ Distributive laws and Koszulness, Ann. Inst. Fourier (Grenoble) 46 (1996), 307-323.

[MSS] M. Markl, S. Shnider, J. Stasheff, Operads in algebra, topology and physics, Mathematical Surveys and Monographs, 96. American Mathematical Society, Providence, RI, 2002.

[M] J. P. May, The geometry of iterated loop spaces, Lectures Notes in Mathematics, Vol. 271, Springer-Verlag, Berlin-New York, 1972.

[Pr] S. Priddy, Koszul resolutions, Trans. Amer. Math. Soc. 152 (1970), 39-60.

[SVO] S. Shnider, D. Van Osdol, Operads as abstract algebras, and the Koszul property, J. Pure Appl. Algebra 143 (1999), 381-407.

[Sm] J. Smith, Operads and algebraic homotopy. II. Suspensions, preprint: math.AT/0102069

[Tr] T. Tradler, Infinity-inner-products on A-infinity-algebras, preprint: math.AT/0108027

Department of Mathematics, University of Chicago, Chicago, IL 60637, U.S.A.

E-mail address: wlgan@math.uchicago.edu 